\title{
Efficacy of Immunohistochemical Staining in Differentiating a Squamous Cell Carcinoma in Poorly Differentiated Rectal Cancer: Two Case Reports
}

\author{
Sairafi Rami, Yoon Dae Han, Mi Jang', Min Soo Cho, Hyuk Hur, Byung Soh Min, Kang Young Lee, \\ Nam Kyu Kim \\ Departments of Surgery and ${ }^{1}$ Clinical Pathology, Yonsei University College of Medicine, Seoul, Korea
}

\begin{abstract}
A rectal carcinoma, including primary an adenosquamous and a squamous cell carcinoma (SCC), is a very rare disease, accounting for $0.025 \%$ to $0.20 \%$ of all large-bowel malignant tumors. Because SCCs have a higher mortality than adenosquamous carcinomas, determining whether the primary rectal cancer exhibits an adenomatous component or a squamous component is important. While differentiating between these 2 components, especially in poorly differentiated rectal cancer, is difficult, specific immunohistochemical stains enable accurate diagnoses. Here, we report the use of immunohistochemical stains to distinguish between the adenomatous and the squamous components in 2 patients with low rectal cancer, a 58-year-old man and a 73-year-old woman, who were initially diagnosed using the histopathologic results for a poorly differentiated carcinoma. These data suggest that using these immunohistochemical stains will help to accurately diagnose the type of rectal cancer, especially for poorly differentiated carcinomas, and will provide important information to determine the proper treatment for the patient.
\end{abstract}

Keywords: Adenosquamous carcinoma; Squamous cell carcinoma; Rectal neoplasms; Immunohistochemistry

\section{INTRODUCTION}

Most rectal malignancies are adenocarcinomas, which are primarily treated surgically, with selective use of neoadjuvant or adjuvant chemo-radiation for more locally advanced disease. Rectal primary adenosquamous carcinoma or squamous cell carcinoma (SCC) is very rare, with an incidence of $0.025 \%$ to $0.20 \%$ among all large bowel malignant tumors [1]. Most cases of rectal SCC are actually anal SCC with proximal extension into the rectum, which is different from primary SCC of the rectum. The estimated incidence of primary rectal SCC is approximately $0.01 \%-0.025 \%$ of

Received: August 3, 2015 • Accepted: March 16, 2016

Correspondence to: Nam Kyu Kim, M.D.

Department of Surgery, Yonsei University College of Medicine, 50 Yonsei-ro, Seodaemun-gu, Seoul 03722, Korea

Tel: +82-2-2228-2105, Fax: +82-2-313-8289

E-mail:namkyuk@yuhs.ac

(C) 2016 The Korean Society of Coloproctology

This is an open-access article distributed under the terms of the Creative Commons Attribution NonCommercial License (http://creativecommons.org/licenses/by-nc/4.0) which permits unrestricted noncommercial use, distribution, and reproduction in any medium, provided the original work is properly cited. all colorectal neoplasms [2]. William et al. [3] established the following criteria to define SCC of rectal origin: (1) metastasis from other organs to the rectum must be ruled out; (2) there is an absence of anal involvement with SCC; and (3) an anal SCC-lined fistula with the rectum must be ruled out. Various treatment options are being used to manage rectal adenosquamous carcinoma or SCC, but there is still difficulty in determining appropriate treatments. While surgery is considered the gold-standard therapy, the combination of radiotherapy and chemotherapy is an effective alternative therapy [4]. Thus, differentiating among adenocarcinoma, adenosquamous carcinoma, and SCC is a critical issue. Using immunohistochemical stains may be an effective method to differentiate these pathologies. A study from Memorial Sloan Kettering Cancer Center showed that immunohistochemical stains are useful in distinguishing between adenosquamous carcinoma and SCC of the rectum, especially in poorly differentiated cancer [5]. Therefore, we describe 2 cases of poorly differentiated rectal cancer, in which various immunohistochemical stains reveal the final pathology containing either an adenomatous component or a squamous component. 
Volume 32, Number 4, 2016

\section{CASE REPORTS}

\section{Case 1}

A 58-year-old man presented with lower rectal swelling, bleeding, and anemia for 3 years. He was a diabetic patient with no family history of colorectal cancer. A colonoscopy revealed a rectal mass $4 \mathrm{~cm}$ above the anal verge (AV), and histopathology from another hospital showed a poorly differentiated carcinoma. His serum carcinoembryonic antigen (CEA) level was $4.06 \mathrm{ng} / \mathrm{mL}$. Pelvic magnetic resonance imaging (MRI) and computed tomography (CT) of the abdomen and the pelvis showed a fungating mass in the lower rectum extending to the anal canal, with multiple lymph nodes (LNs) in the perirectal and right pelvic side wall without any other evidence of distant metastasis (Fig. 1A). Whole-body positron emission tomography-CT showed intense fluorodeoxyglucose uptake in the LNs at the perirectal and both iliac chains without any suggestion of distant metastasis.

To differentiate the type of cancer, the patient underwent re-biopsy. This revealed a poorly differentiated carcinoma, suggestive of a squamous-cell type. Therefore, additional immunohistochemical staining and a special stain analysis were performed. From the stains, p40 was positive and CK20, CEA, and HMB45 were negative in the tumor cells. Based on the diagnosis of a SCC, the patient started concurrent chemo-radiotherapy (CCRT) for 6 weeks, based on a regimen of fluorouracil and mitomycin $\mathrm{C}$ with 50.4 Gy. One month after CCRT, follow-up rectal MRI and abdominal and pelvic CT were performed. The scans showed a reduction in the size of the pedunculated mass protruding into the anal canal with no extramural extension. Also noted was a reduction in the sizes of the perirectal and the right iliac LNs (Fig. 1B). However, the patient still presented with a prolapsing anal mass (Fig. 1C).

After a multidisciplinary team approach, the patient underwent excision of the prolapsed lesion to confirm the pathology, which showed no residual tumor with nonspecific inflammation. Two months later, rectal MRI showed a new $8.4 \times 3.1-\mathrm{cm}$ lobulating mass that involved the entire anal canal and low rectum and was suspected to be a recurrent tumor. A rebiopsy was performed, and the pathology report showed a poorly differentiated carcinoma expressing p63 and p40, consistent with a SCC, while CDX2 and HMB45 were negative in the tumor cells (Table 1). The patient had an abdominoperineal resection, and the pathology report showed Mandard grade IV regression with a poorly differentiated carcinoma and squamous differentiation, classified as ypT3N0M0. All of the margins were free, but lymphovascular invasion (LVI) and perineural invasion were seen. The permanent immunohistochemical staining results showed positive $\mathrm{p} 40$ with weakly positive p63, and negative CDX2 staining (Fig. 2). The pa-

Table 1. Results of stains for cases 1 and 2

\begin{tabular}{llc}
\hline Case & Stain & Result \\
\hline 1 & p40 & + \\
& p63 & + \\
& CK20 & - \\
& CEA & - \\
& CDX2 & - \\
& HMB45 & - \\
& p40 & + \\
& p63 & + \\
& CEA & + \\
& CK20 & + \\
& CDX2 & - \\
& D-PAS & + \\
\hline
\end{tabular}
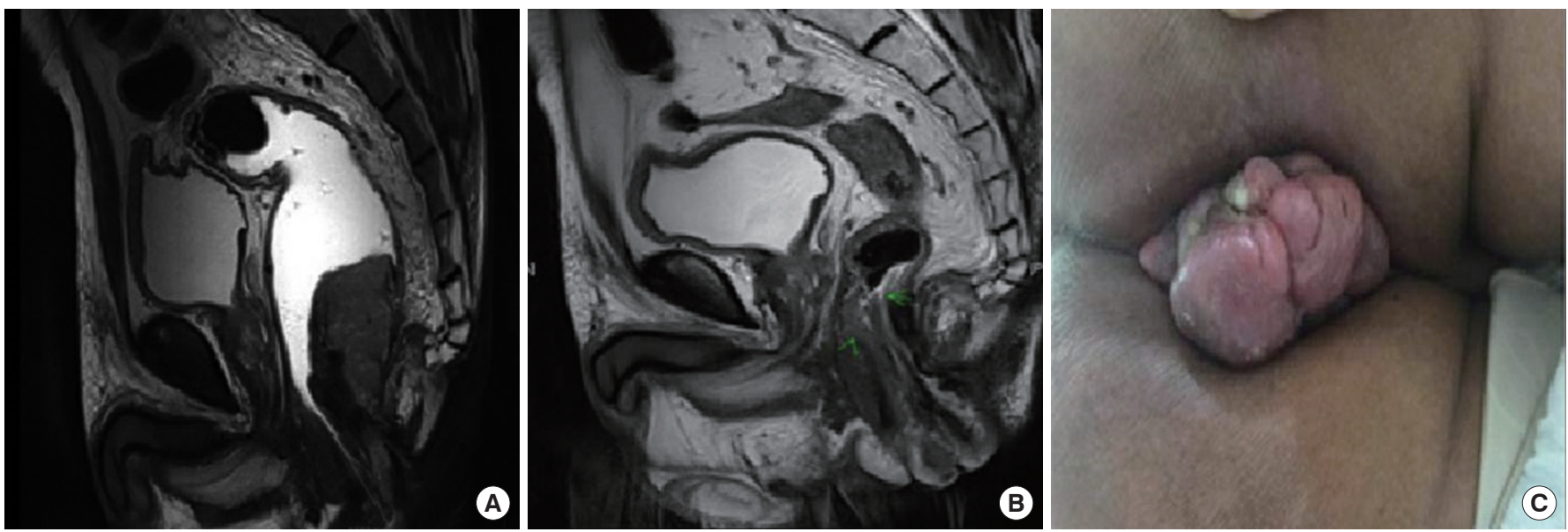

Fig. 1. (A) Pelvic magnetic resonance imaging (MRI) revealed a $6.1-\mathrm{cm}$ fungating mass involving the lower rectum $(4 \mathrm{~cm}$ from the anal verge). Multiple regional lymph node metastases were suspected. (B) Post concurrent chemo-radiotherapy MRI shows a decrease in the size of the pedunculated mass protruding into the anal canal (from $6 \mathrm{~cm}$ to $4.7 \mathrm{~cm}$ in size) with no extramural extension. (C) Encircled prolapsed anal mass. 

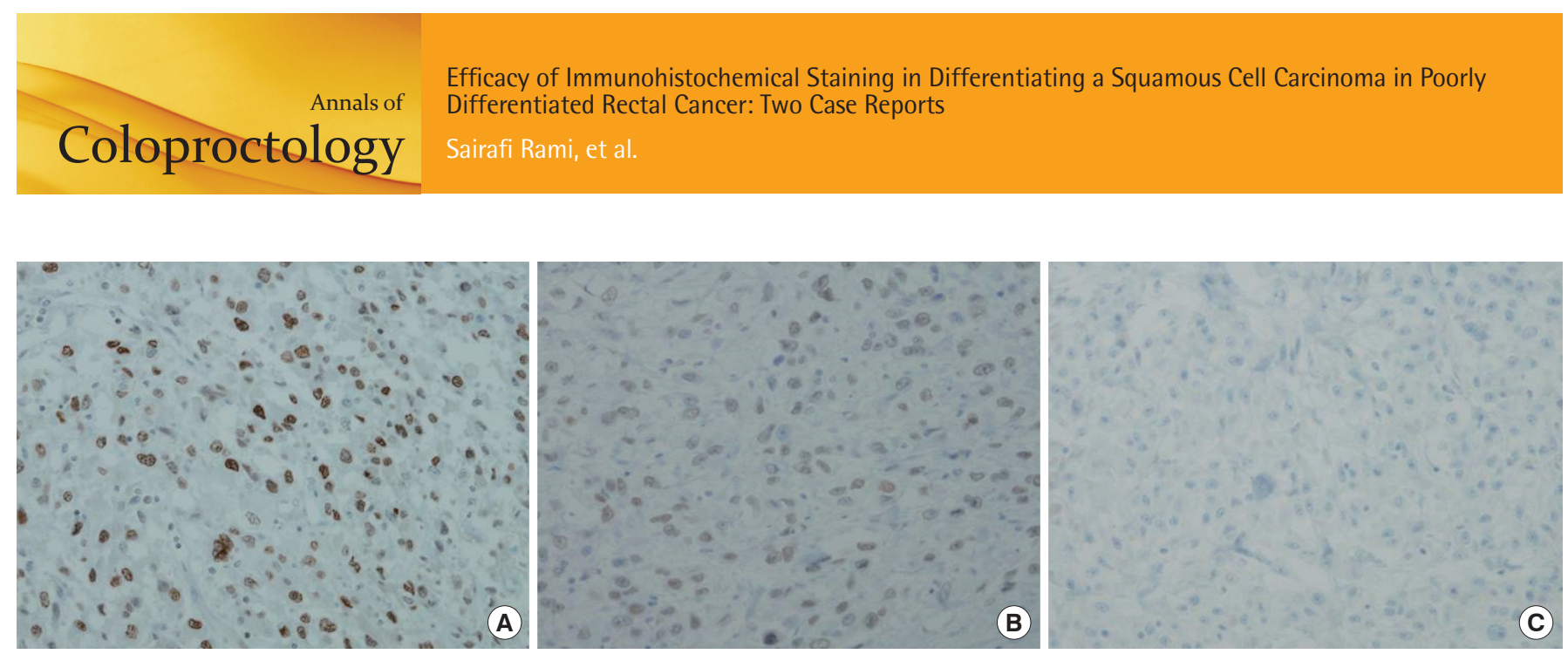

Fig. 2. (A) Positive in tumor cells showing squamous differentiation $(\mathrm{P} 40, \times 200)$, (B) weakly positive in tumor cells showing squamous differentiation $(\mathrm{P} 63, \times 200),(\mathrm{C})$ negative in tumor cells showing squamous differentiation $(\mathrm{CDX} 2, \times 200)$.
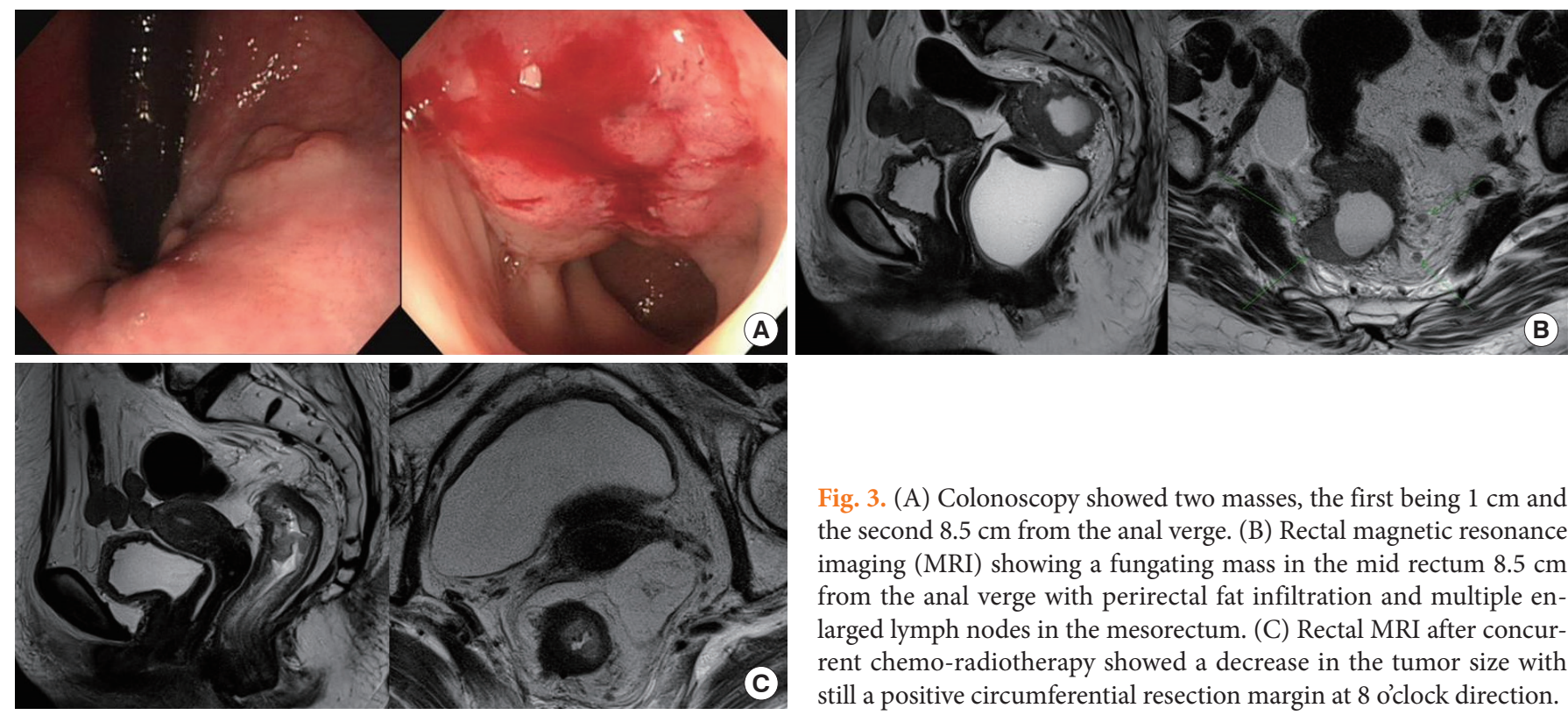

Fig. 3. (A) Colonoscopy showed two masses, the first being $1 \mathrm{~cm}$ and the second $8.5 \mathrm{~cm}$ from the anal verge. (B) Rectal magnetic resonance imaging (MRI) showing a fungating mass in the mid rectum $8.5 \mathrm{~cm}$ from the anal verge with perirectal fat infiltration and multiple enlarged lymph nodes in the mesorectum. (C) Rectal MRI after concurrent chemo-radiotherapy showed a decrease in the tumor size with still a positive circumferential resection margin at 8 oclock direction.

tient was administered 5-fluorouracil and cisplatin chemotherapy after surgery, and he is now on his third chemotherapy cycle.

\section{Case 2}

A 73-year-old woman presented with a 3-month history of changes in stool habit and stool caliber. She had a positive stool occult blood test, and her serum CEA level was $1.69 \mathrm{ng} / \mathrm{mL}$. Colonoscopy showed 2 masses; one was $1 \mathrm{~cm}$ from the $\mathrm{AV}$, and the other was $8.5 \mathrm{~cm}$ from the AV (Fig. 3A). The pathology report from another hospital indicated a diagnosis of a poorly differentiated carcinoma. Rectal MRI and CT of the abdomen and the pelvis showed a fungating mass in the midrectum, with perirectal fat infiltration and multiple enlarged LNs in the mesorectum. No pelvic side wall or para-aortic lymphadenopathy was indicated (Fig. 3B), and chest CT showed no evidence of intrathoracic metastasis. Rebiopsy was performed for suspected lesions, and the pathology report showed a fragment of scattered tumor cells with p63-positive nuclei, consistent with a SCC.

Because the disease was considered as T1 anal cancer and T3 rectal cancer positive for a circumferential resection margin (CRM), a multidisciplinary team decided to start neoadjuvant CCRT. After 4 weeks of Xeloda-based 50.4-Gy CCRT, follow-up rectal MRI and CT of the abdomen and the pelvis showed decreased tumor volume with mesorectal infiltration, but the scans were still positive for CRM at the 8 o'clock position with no significant LNs in the pelvic cavity or evidence of distant metastasis (Fig. 3C). Ten weeks after CCRT, the patient underwent a laparoscopic-assisted low anterior resection with double stapling for rectal cancer and a transanal excision for anal cancer (Fig. 4). The pathological diagnosis after surgery showed post-CCRT status with fibrosis outgrowing the residual cancer with a main diagnosis of a poorly differentiated adenosquamous carcinoma. LVI was positive, and an ulcerative $2.5 \times 1.5-\mathrm{cm}$ mass with a classification of ypT3N0M0 was seen. 

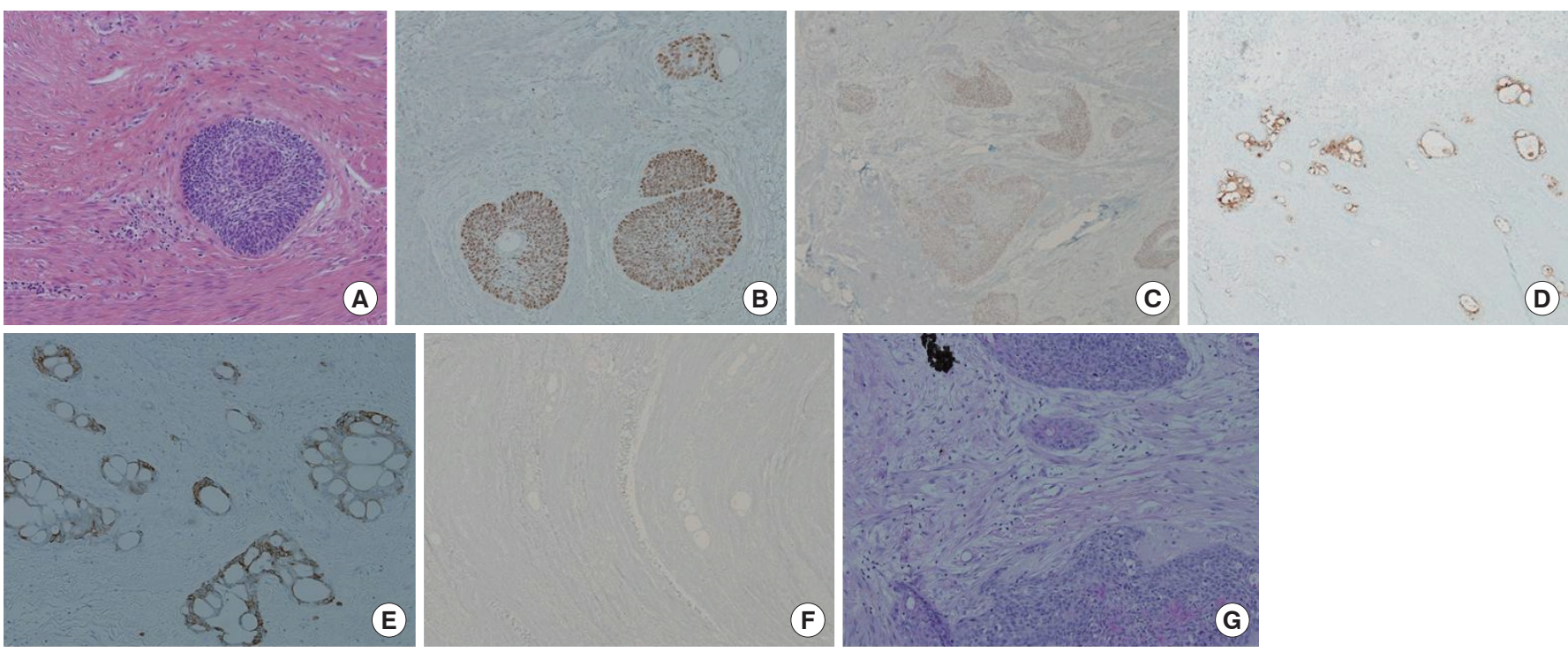

Fig. 4. (A) H\&E, $\times 100$, (B) positive in squamous cell carcinomatous component $(\mathrm{P} 40, \times 100)$, (C) positive in squamous cell carcinomatous component $(\mathrm{P} 63, \times 40),(\mathrm{D})$ positive in adenocarcinomatous component $(\mathrm{CEA}, \times 40)$, (E) positive in adenocarcinomatous component $(\mathrm{CK} 20, \times 100)$, (F) negative in tumor cells $(\mathrm{CDX} 2, \times 40)$, and $(\mathrm{G})$ focal mucin deposits in tumor cells (D-PAS, $\times 100)$.

Table 2. Immunohistochemical stain markers used to distinguish between the adenomatous and squamous components

\begin{tabular}{|c|c|c|c|}
\hline Marker & Stain & General & Uses \\
\hline p40 & deltaNp63 & p63 isoforms & $\begin{array}{l}\text { Classify nonsmall cell lung carcinoma: } \\
\text {-Adenocarcinoma (deltaNp63 -ve or }<2 \% \text { of cells with weak staining) } \\
\text {-Squamous cell carcinoma (deltaNp63+ve [strong] and }>50 \% \text { of cells) } \\
\text {-Adenosquamous (deltaNp63+ve [strong] and }<50 \% \text { of cells) }[15,16]\end{array}$ \\
\hline CK20 & Cytokeratin20 & $\begin{array}{l}\text { Epithelial marker with restricted } \\
\text { expression compared with } \\
\text { CK7 }\end{array}$ & $\begin{array}{l}\text {-CK7-/CK20+: carcinoma of the colon, particularly early stage [17] } \\
\text {-CK20 is less sensitive for poorly differentiated colonic carcinomas [18] } \\
\text {-Primary mucinous tumors of lower gastrointestinal tract [19] } \\
\text {-To help distinguish colon carcinomas (80\% are CK20+) at biopsy [20] } \\
\text {-To distinguish anal carcinomas (CK7+/CK20-) from downward growth of colorectal } \\
\text { carcinomas [21] }\end{array}$ \\
\hline CEA & CD66e & & $\begin{array}{l}\text { Colorectal carcinoma: } \\
\text {-Monitor serum levels (elevated in 72\%-97\%) to detect recurrence [22] } \\
\text {-Elevated preoperative serum levels are a poor prognostic factor [23] } \\
\text {-Elevated levels also present in cirrhosis, biliary obstruction, hepatitis, inflammatory } \\
\text { bowel disease, smokers and post-surgical bowel sequestration with mucocele [24] }\end{array}$ \\
\hline HMB45 & Human melanoma black 45 & Pmel 17 & $\begin{array}{l}\text {-Reacts against an antigen present in melanocyte tumors; } 92 \% \text { sensitivity when used to } \\
\text { identify melanomas [25] }\end{array}$ \\
\hline p63 & Tap63, deltaNp63 & Member of p53 gene family & $\begin{array}{l}\text {-Determines squamous differentiation (p63+) for hematoxylin-eosin or cytology cases as } \\
\text { part of a panel [26], but p63 rarely stains an adenocarcinoma [27] }\end{array}$ \\
\hline CDX2 & $\begin{array}{l}\text { Homeobox gene that encodes a } \\
\text { nuclear transcription factor } \\
\text { critical for intestinal embry- } \\
\text { onic development }\end{array}$ & & $\begin{array}{l}\text {-Fairly specific marker of gastrointestinal origin for adenocarcinomas, but also stains } \\
\text { selected adenocarcinomas of other sites [28] } \\
\text {-Used to determine the origin of a metastatic adenocarcinoma as part of the panel [29] } \\
\text {-Distinguish primary and secondary colorectal adenocarcinomas [30] }\end{array}$ \\
\hline
\end{tabular}

The immunohistochemical stain results showed positive for $\mathrm{p} 40$ and p63, suggesting a squamous cell carcinomatous component. Additionally, CEA and CK20 were positive for the adenocarcinomatous component, and CDX2 was negative in the tumor cells.
D-PAS stain revealed focal mucin deposits in the tumor cells (Table 1). A follow-up CT scan of the abdomen and the pelvis one month after the surgery showed no evidence of tumor recurrence or metastasis, and the patient started on 4 cycles of FP (5-fluoro- 
uracil and cisplatin) chemotherapy. The FP chemotherapy has been completed, and the patient is being followed up at an outpatient clinic without any recurrence.

\section{DISCUSSION}

Colorectal cancer is a common disease that usually presents with an adenocarcinoma histology. However, only a few reports in the literature are concerned with colorectal SCCs. The etiology of a SCC is uncertain. According to Balfour, a true SCC of the colon and rectum does not exist, and this disease might represent metastasis from other sites or squamous degeneration of a pre-existing adenocarcinoma. However, this opinion is not widely accepted [6]. Several other theories have attempted to explain the histopathogenesis of these tumors. These theories include (1) the presence of embryologic nests of ectodermal cells in the colonic mucosa; (2) the occurrence of squamous metaplasia of the intestinal mucosa, specifically in colonic adenomas; (3) the capability of pluripotent stem cells of endodermal origin to transform into an adenocarcinoma, a SCC, or both; and (4) possible cellular degeneration within an existing adenocarcinoma secondary to an abnormal mucosa stimulus such as ulcerative colitis or radiation [1, 7]. Because defining an adenocarcinoma, an adenosquamous carcinoma or a SCC, is difficult in poorly differentiated cancer, several immunohistochemical markers are used to distinguish squamous-cell disease from an adenocarcinoma (Table 2).

Hematoxylin-eosin (H\&E) staining is first applied to detect whether the adenomatous morphology is present. Following the stain analysis, if clarifying the type is still difficult, extra immunohistochemical stains should be used. In this report, both cases required extra staining to confirm the diagnosis because the primary result was only a poorly differentiated carcinoma. p40 and p63 are important markers for discriminating squamous cell components from adenocarcinoma components because these markers are positive in SCCs. p63 is p53 homolog nonspecific antibody, subdivided by TAp63 and deltaNp63, which is called p40, so the expression of deltaNp63, as well as that of p63, is compared with both the clinical and the pathological factors and prognosis [8].

In the first case, we also stained with HMB45 to distinguish this cancer, which presented very poor differentiation in the anus, from a malignant melanoma. Furthermore, because H\&E staining suggested an adenosquamous carcinoma in the second case study, D-PAS, which can be derived from an adenocarcinoma, was stained only in the second case. In both cases, additional staining for the expressions of CEA, CK20, and CDX2 was performed. These markers are positive in an adenocarcinoma, possibly aiding in the discrimination of the adenomatous component from the primary cancer.

Early discrimination of a rectal adenocarcinoma from an adenosquamous carcinoma or a SCC is critical because of differences among prognoses. For instance, the prognosis for a rectal SCC is worse than that of other parts of the colon [9]. A preoperative diagnosis is difficult because the clinical presentation of a SCC is similar to that of an adenocarcinoma; symptoms include abdominal pain, anemia, weight loss, rectal bleeding, and tenesmus [10, 11]. Thus, with the help of immunohistochemical staining analysis, discriminating among a rectal adenocarcinoma, adenosquamous disease, and a SCC is possible. The squamous epithelial components have more invasive tendencies than the glandular components [7], and the survival rates are lower in the epithelial components $[10,11]$. Furthermore, in cases of LN metastasis, the prognosis for an adenosquamous carcinoma or a SCC is much worse than that for an adenocarcinoma alone [6]. The 5-year survival rate for a node-positive adenosquamous carcinoma or a SCC is $23 \%$ compared with $85 \%$ for node-negative cases. The overall 5-year survival rate for an adenosquamous carcinoma or a SCC is $31 \%$ compared with $66 \%$ for an adenocarcinoma [12].

Overall, surgical resection is traditionally considered the most effective treatment not only to cure the patient but also to confirm the pathology. Some researchers have suggested that chemoradiation may be an effective treatment, or even consider it a definitive treatment, reserving surgery for patients for whom chemoradiation treatment has failed $[10,13]$. The low acute toxicity of chemoradiation and the rare incidence of long-term toxicity, reported as symptomatic rectal stricture, are reasons to consider treatments other than surgery, which has the risks of morbidity and mortality [14]. Thus, chemotherapy or radiotherapy should be considered, especially in node-positive cases, although further investigation is needed regarding whether to treat patients with chemotherapy or radiotherapy alone or to treat them with a combination of these therapies.

In summary, compared to an adenocarcinoma, an adenosquamous carcinoma and a SCC exhibit both poor prognoses and difficulties in determining treatment plans. Using several immunohistochemical stains, we were able to distinguish squamous components from adenomatous components, especially in patients with poorly differentiated cancer. By differentiating between an adenosquamous carcinoma and a SCC, physicians may employ more effective treatments for these rare malignancies.

\section{CONFLICT OF INTEREST}

No potential conflict of interest relevant to this article was reported.

\section{REFERENCES}

1. Schlegel RD, Dehni N, Cravino AT, Tiret E, Prevot S, Waisman $\mathrm{HJ}$, et al. Primary adenosquamous carcinoma of the rectum. Report of 4 cases and review of the literature. Colorectal Dis 2001;3: 201-3.

2. Scaringi S, Bisogni D, Messerini L, Bechi P. Squamous cell carcinoma of the middle rectum: report of a case and literature over- 
view. Int J Surg Case Rep 2015;7C:127-9.

3. Williams GT, Blackshaw AJ, Morson BC. Squamous carcinoma of the colorectum and its genesis. J Pathol 1979;129:139-47.

4. Rasheed S, Yap T, Zia A, McDonald PJ, Glynne-Jones R. Chemoradiotherapy: an alternative to surgery for squamous cell carcinoma of the rectum--report of six patients and literature review. Colorectal Dis 2009;11:191-7.

5. Nahas CS, Shia J, Joseph R, Schrag D, Minsky BD, Weiser MR, et al. Squamous-cell carcinoma of the rectum: a rare but curable tumor. Dis Colon Rectum 2007;50:1393-400.

6. Dong Y, Wang J, Ma H, Zhou H, Lu G, Zhou X. Primary adenosquamous carcinoma of the colon: report of five cases. Surg Today 2009;39:619-23.

7. Frizelle FA, Hobday KS, Batts KP, Nelson H. Adenosquamous and squamous carcinoma of the colon and upper rectum: a clinical and histopathologic study. Dis Colon Rectum 2001;44:341-6.

8. Morita M, Uramoto H, Nakata S, Ono K, Sugaya M, Yoshimatsu T, et al. Expression of deltaNp63 in squamous cell carcinoma of the esophagus. Anticancer Res 2005;25:3533-9.

9. Dyson T, Draganov PV. Squamous cell cancer of the rectum. World J Gastroenterol 2009;15:4380-6.

10. Schneider TA 2nd, Birkett DH, Vernava AM 3rd. Primary adenosquamous and squamous cell carcinoma of the colon and rectum. Int J Colorectal Dis 1992;7:144-7.

11. Petrelli NJ, Valle AA, Weber TK, Rodriguez-Bigas M. Adenosquamous carcinoma of the colon and rectum. Dis Colon Rectum 1996;39:1265-8.

12. Jemal A, Siegel R, Ward E, Murray T, Xu J, Thun MJ. Cancer statistics, 2007. CA Cancer J Clin 2007;57:43-66.

13. Iannacone E, Dionisi F, Musio D, Caiazzo R, Raffetto N, Banelli E. Chemoradiation as definitive treatment for primary squamous cell cancer of the rectum. World J Radiol 2010;2:329-33.

14. Brammer RD, Taniere P, Radley S. Metachronous squamous-cell carcinoma of the colon and treatment of rectal squamous carcinoma with chemoradiotherapy. Colorectal Dis 2009;11:219-20.

15. Pelosi G, Fabbri A, Bianchi F, Maisonneuve P, Rossi G, Barbareschi M, et al. $\triangle \mathrm{Np} 63$ (p40) and thyroid transcription factor-1 immunoreactivity on small biopsies or cellblocks for typing nonsmall cell lung cancer: a novel two-hit, sparing-material approach. J Thorac Oncol 2012;7:281-90.

16. Nonaka D. A study of $\Delta \mathrm{Np} 63$ expression in lung non-small cell carcinomas. Am J Surg Pathol 2012;36:895-9.

17. Hernandez BY, Frierson HF, Moskaluk CA, Li YJ, Clegg L, Cote $\mathrm{TR}$, et al. CK20 and CK7 protein expression in colorectal cancer: demonstration of the utility of a population-based tissue microarray. Hum Pathol 2005;36:275-81.

18. Wu CY, Lee WH, Wang JY, Chiang H, Chang JL, Tsai WC, et al. Tissue microarray-determined expression profiles of cyclooxygenase-2 in colorectal adenocarcinoma: association with clinico- pathological parameters. Chin J Physiol 2006;49:298-304.

19. Vang R, Gown AM, Barry TS, Wheeler DT, Yemelyanova A, Seidman JD, et al. Cytokeratins 7 and 20 in primary and secondary mucinous tumors of the ovary: analysis of coordinate immunohistochemical expression profiles and staining distribution in 179 cases. Am J Surg Pathol 2006;30:1130-9.

20. Owens CL, Epstein JI, Netto GJ. Distinguishing prostatic from colorectal adenocarcinoma on biopsy samples: the role of morphology and immunohistochemistry. Arch Pathol Lab Med 2007; 131:599-603.

21. Ramalingam P, Hart WR, Goldblum JR. Cytokeratin subset immunostaining in rectal adenocarcinoma and normal anal glands. Arch Pathol Lab Med 2001;125:1074-7.

22. Fernandes LC, Kim SB, Saad SS, Matos D. Value of carcinoembryonic antigen and cytokeratins for the detection of recurrent disease following curative resection of colorectal cancer. World J Gastroenterol 2006;12:3891-4.

23. Louhimo J, Carpelan-Holmström M, Alfthan H, Stenman UH, Järvinen HJ, Haglund C. Serum HCG beta, CA 72-4 and CEA are independent prognostic factors in colorectal cancer. Int J Cancer 2002;101:545-8.

24. Haber MM, Leon ME, Bakker JE, Nagle D. Carcinoembryonic antigen elevation due to bowel sequestration with mucocele formation following colonic resection. Arch Pathol Lab Med 2003; 127:1376-9.

25. Mahmood MN, Lee MW, Linden MD, Nathanson SD, Hornyak TJ, Zarbo RJ. Diagnostic value of HMB-45 and anti-Melan A staining of sentinel lymph nodes with isolated positive cells. Mod Pathol 2002;15:1288-93.

26. Ocque R, Tochigi N, Ohori NP, Dacic S. Usefulness of immunohistochemical and histochemical studies in the classification of lung adenocarcinoma and squamous cell carcinoma in cytologic specimens. Am J Clin Pathol 2011;136:81-7.

27. Mukhopadhyay S, Katzenstein AL. Subclassification of non-small cell lung carcinomas lacking morphologic differentiation on biopsy specimens: utility of an immunohistochemical panel containing TTF-1, napsin A, p63, and CK5/6. Am J Surg Pathol 2011; 35:15-25.

28. Werling RW, Yaziji H, Bacchi CE, Gown AM. CDX2, a highly sensitive and specific marker of adenocarcinomas of intestinal origin: an immunohistochemical survey of 476 primary and metastatic carcinomas. Am J Surg Pathol 2003;27:303-10.

29. Park SY, Kim BH, Kim JH, Lee S, Kang GH. Panels of immunohistochemical markers help determine primary sites of metastatic adenocarcinoma. Arch Pathol Lab Med 2007;131:1561-7.

30. Groisman GM, Bernheim J, Halpern M, Brazowsky E, Meir A. Expression of the intestinal marker $\mathrm{Cdx} 2$ in secondary adenocarcinomas of the colorectum. Arch Pathol Lab Med 2005;129:920-3. 